\title{
Effects of exercise on mitochondrial DNA content in skeletal muscle of patients with COPD
}

\author{
Luis Puente-Maestu, ${ }^{1,2}$ Alberto Lázaro, ${ }^{3}$ Alberto Tejedor, ${ }^{3}$ Sonia Camaño, ${ }^{3}$ \\ Marta Fuentes, ${ }^{1,2}$ Miguel Cuervo, ${ }^{4}$ Beatriz Oláiz Navarro, ${ }^{5}$ Alvar Agusti $i^{6,7^{\prime}}$
}

${ }^{1}$ Servicio de Neumología, Hospital General Universitario Gregorio Marañón, Madrid,

Spain

${ }^{2}$ Universidad Complutense de Madrid, Madrid, Spain

${ }^{3}$ Laboratorio de Nefrología Experimental, Hospital General Universitario Gregorio Marañón, Universidad Complutense de

Madrid, Madrid, Spain

${ }^{4}$ Servicio de Traumatología,

Hospital General Universitario

Gregorio Marañón; Universidad

Complutense de Madrid,

Madrid, Spain

${ }^{5}$ Servicio de Cirugía de Tórax, Hospital General Universitario de Getafe, Madrid, Spain

${ }^{6}$ Institut del Torax, Hospital Clínic, IDIBAPS, Barcelona,

Spain

${ }^{7}$ CIBER Enfermedades

Respiratorias (CIBERES) y

Fundación Caubet-Cimera,

Mallorca, Spain

\section{Correspondence to}

Luis Puente-Maestu, Servicio de Neumología, Hospital General Universitario Gregorio Marañón, c/Doctor Ezquerdo 46, 28007

Madrid, Spain;

Ipuente.hgugm@salud.madrid.org

Received 7 October 2010

Accepted 27 October 2010

Published Online First

20 November 2010

\begin{abstract}
Background Exhausting exercise reduces the mitochondrial DNA (mtDNA) content in the skeletal muscle of healthy subjects due to oxidative damage. Since patients with chronic obstructive pulmonary disease (COPD) suffer enhanced oxidative stress during exercise, it was hypothesised that the mtDNA content will be further reduced.
\end{abstract}

Objective To investigate the effects of exercise above and below the lactate threshold (LT) on the mtDNA content of skeletal muscle of patients with COPD.

Methods Eleven patients with COPD (67 \pm 8 years; forced expiratory volume in $1 \mathrm{~s}$ (FEV 1 ) $45 \pm 8 \%$ ref) and 10 healthy controls (66 \pm 4 years; FEV $190 \pm 7 \%$ ref) cycled 45 min above LT (65\% peak oxygen uptake $\left(V^{\prime} 0_{2}\right.$ peak) and another 7 patients (65 \pm 6 years; $\mathrm{FEV}_{1} 50 \pm 4 \%$ ref) and 7 controls ( $56 \pm 9$ years; $\mathrm{FEV}_{1} 92 \pm 6 \%$ ref) cycled 45 min below their LT ( $50 \% \mathrm{~V}^{\prime} \mathrm{o}_{2}$ peak). Biopsies from the vastus lateralis muscle were obtained before exercise, immediately after and $1 \mathrm{~h}, 1$ day and 1 week later to determine by PCR the mtDNA/nuclear DNA (nDNA) ratio (a marker of mtDNA content) and the expression of the peroxisome proliferator-activated receptor- $\gamma$ coactivator- $1 \alpha$ (PGC-1 $\alpha$ ) mRNA and the amount of reactive oxygen species produced during exercise was estimated from total $\mathrm{V}^{\prime} \mathrm{O}_{2}$.

Results Skeletal muscle mtDNA/nDNA fell significantly after exercise above the LT both in controls and in patients with COPD, but the changes were greater in those with COPD. These changes correlated with production of reactive oxygen species, increases in manganese superoxide dismutase and PGC- $1 \alpha$ mRNA and returned to baseline values 1 week later. This pattern of response was also observed, albeit minimised, in patients exercising below the LT.

Conclusions In patients with COPD, exercise enhances the decrease in mtDNA content of skeletal muscle and the expression of PGC-1 $\alpha$ mRNA seen in healthy subjects, probably due to oxidative stress.

\section{INTRODUCTION}

Exercise limitation is a frequent complaint of patients with chronic obstructive pulmonary disease (COPD) and a major precipitator of their poor health status. Several cellular and molecular alterations that can contribute to exercise limitation have been described in the skeletal muscle of these patients. ${ }^{1-7}$ Our group has recently identified several mitochondrial abnormalities, including malfunction of the electron transport chain, abnormal kinetics of the mitochondrion permeability transition pore and excessive production of reactive oxygen species (ROS), ${ }^{8} 9$ that can also contribute to exercise limitation and skeletal muscle dysfunction in COPD

In healthy subjects, fatiguing exercise causes a reduction in the mitochondrial DNA (mtDNA) content of skeletal muscle due to oxidative damage whereas low-intensity non-fatiguing exercise does not. ${ }^{10}$ This has not been investigated in patients with COPD but, because mitochondrial ROS production is augmented in such patients, ${ }^{8} 11-13$ we hypothesised that the fall in mtDNA content reported in healthy subjects ${ }^{10}$ will be enhanced in patients with COPD, particularly during fatiguing exercise above their lactate threshold (LT). Accordingly, this study sought to compare the dynamic changes induced by two types of exercise protocols (above and below the LT) in mtDNA content of the vastus lateralis muscle in patients with COPD and healthy controls. To get an insight into the potential molecular mechanisms, we also investigated the amount of ROS produced during exercise, the dynamic changes in the activity of manganese superoxide dismutase (MnSOD; EC 1.15.1.1), a major antioxidant enzyme, and of the expression of peroxisome proliferator-activated receptor- $\gamma$ coactivator- $1 \alpha$ messenger RNA (PGC- $1 \alpha$ mRNA), a master regulator of mitochondrial biogenesis ${ }^{14}$ which appears to be abnormal in COPD. ${ }^{6} \mathrm{~A}$ better understanding of mitochondrial changes induced by exercise in the skeletal muscle of patients with COPD may be helpful for the design of improved rehabilitation strategies.

\section{METHODS \\ Population}

Patients with COPD (diagnosis according to the GOLD guidelines ${ }^{15}$ ) had moderate to severe airflow limitation (forced expiratory volume in $1 \mathrm{~s}\left(\mathrm{FEV}_{1}\right)$ $<65 \%$ reference value), declared to have ceased smoking at least 6 months before enrolling, had not participated in a rehabilitation programme before and had no contraindication for exercise testing. Controls were non-smoking subjects with normal aerobic capacity and resting pulmonary function without any obvious lung, cardiac or other major disease after reviewing their medical information.

\section{Study design}

This is a prospective and controlled study. To determine peak oxygen uptake $\left(\mathrm{V}^{\prime} \mathrm{O}_{2}\right.$ peak), all participants performed an incremental $(20 \mathrm{~W} /$ min) symptom-limited exercise test on a cycle ergometer (ER-900, Jaeger, Hochberg, Germany) with continuous monitoring of oxygen saturation (Nellcor N-180, Pleasanton, California, USA) and 
breath-by-breath measurements of ventilation and pulmonary gas exchange (Quark- $b^{2}$ system, Cosmed, Rome, Italy). Those who developed severe hypoxaemia $\left(\mathrm{SaO}_{2}<87 \%\right)$ during the incremental test were excluded from further investigations. Then, 1-2 weeks later, the effects of two constant exercise protocols, above and below the LT, were investigated. The former entailed $3 \mathrm{~min}$ resting, $3 \mathrm{~min}$ unloaded pedalling and a total of $45 \mathrm{~min}$ pedalling $(60 \mathrm{rpm})$ at $65 \%$ of $\mathrm{V}^{\prime} \mathrm{O}_{2}$ peak whereas the latter entailed pedalling ( $60 \mathrm{rpm})$ at $50 \%$ of $\mathrm{V}^{\prime} \mathrm{O}_{2}$ peak during 45 min. An arterial blood sample was anaerobically drawn immediately after exercise and analysed for blood gases and lactate concentration (Synthesis 1740, Instrumentation Laboratory, Lexington, Massachusetts, USA). Different subjects participated in the two exercise protocols (table 1) because of the high number of muscle biopsies needed (see limitations).

\section{Patient characterisation}

Spirometry was determined in the seated position with a Masterscope-PFT system (VYASIS, Hochberg, Germany); reported values correspond to those measured 15 min after the inhalation of $200 \mu \mathrm{g}$ salbutamol. Fat-free mass was measured by bioelectrical impedance (Bodystat 1500, Bodystat Ltd, Douglas, Isle of Man, UK).

\section{Muscle biopsies}

Biopsies from the vastus lateralis muscle were obtained with an 18G Tru-Cut biopsy needle (Cardinal Health-España, Madrid,
Spain) 2-7 days before the exercise test, immediately after it, and $1 \mathrm{~h}, 24 \mathrm{~h}$ and 1 week later. Two patients with COPD and three controls refused to be biopsied 1 week after exercise. The biopsy specimens were immediately frozen and stored at $-80^{\circ} \mathrm{C}$ until analysis.

\section{PCR measurements}

To estimate the mDNA content we quantified the mtDNA to nuclear DNA (nDNA) ratio (mtDNA/nDNA). Total DNA was extracted (OIAGEN GmbH, Hilden, Germany) and quantitated spectrophotometrically. The mtDNA content was measured by PCR (ABI Prisma 7700, Perkin-Elmer, California, USA) and corrected by the simultaneous measurement of a single copy nuclear RNAseP gene. Primers used for the analysis of mtDNA were mt806F (5'-CCACGGGAAACAGCAGTGATT-3') and mt929R (5'-CTATTGACTTGGGTTAAT CGTGTGA-3'). The TaqMan probe was labelled at the $5^{\prime}$ end with the fluorescent dye FAM (5'TGCCAGCCACCGCG-3'). To quantify nDNA we used a commercial kit (PDARs RNAseP; Applied Biosystems, Carlsbad, CA, USA) and a nDNA-specific fluorescent probe labelled internally using the fluorescent dye VIC. PCR conditions were $2 \mathrm{~min}$ at $50^{\circ} \mathrm{C}$ and $10 \mathrm{~min}$ at $95^{\circ} \mathrm{C}$, followed by 40 cycles of $15 \mathrm{~s}$ of denaturation at $95^{\circ} \mathrm{C}$ and $60 \mathrm{~s}$ of annealing/ extension at $60^{\circ} \mathrm{C}$. Data are expressed as the mean of three measurements.

The amount of PGC- $1 \alpha \mathrm{mRNA}$ was also quantified by PCR (ABI Prisma 7700, Perkin-Elmer, Foster City, California, USA). The

Table 1 Characteristics of the study population

\begin{tabular}{|c|c|c|c|c|}
\hline & \multicolumn{2}{|l|}{ Above LT } & \multicolumn{2}{|l|}{ Below LT } \\
\hline & Controls $(n=10)$ & COPD $(n=11)$ & Controls $(n=7)$ & COPD $(n=7)$ \\
\hline \multicolumn{5}{|l|}{ Clinical data } \\
\hline Age (years) & $66.5 \pm 4.3$ & $67.2 \pm 8.4$ & $56.7 \pm 8.9$ & $65.0 \pm 6.5$ \\
\hline FFMI $\left(\mathrm{kg} / \mathrm{m}^{2}\right)$ & $21.0 \pm 2.6$ & $19.2 \pm 1.4$ & $22.4 \pm 5.3$ & $19.3 \pm 1.8$ \\
\hline \multicolumn{5}{|l|}{ Lung function and peak exercise data } \\
\hline $\mathrm{FEV}_{1}(\mathrm{I})$ & $2.9 \pm 0.4$ & $1.4 \pm 0.3^{* * *}$ & $2.8 \pm 0.5$ & $1.6 \pm 0.3^{* * *}$ \\
\hline $\mathrm{PaO}_{2}$ at rest $(\mathrm{mm} \mathrm{Hg})$ & $77 \pm 7$ & $67 \pm 7^{* *}$ & $83 \pm 3$ & $66 \pm 5^{* * *}$ \\
\hline $\mathrm{V}^{\prime} \mathrm{O}_{2}$ peak $(\mathrm{I} / \mathrm{min})$ & $1.67 \pm 0.23$ & $1.23 \pm 0.18^{* *}$ & $1.73 \pm 0.34$ & $1.34 \pm 0.29^{*}$ \\
\hline $\mathrm{V}^{\prime} \mathrm{O}_{2}$ peak (\% ref) & $91 \pm 3.3$ & $68 \pm 10.4^{* * *}$ & $90 \pm 2.3$ & $71 \pm 11.9^{* *}$ \\
\hline$V^{\prime} 0_{2}$ at $L T(1 / \mathrm{min})$ & $1.03 \pm 0.13$ & $0.73 \pm 0.15^{* *}$ & $0.82 \pm 0.10$ & $1.06 \pm 0.26^{* *}$ \\
\hline Work rate peak (W) & $132 \pm 23$ & $88 \pm 17^{* *}$ & $143 \pm 35$ & $99 \pm 27^{*}$ \\
\hline \multicolumn{5}{|l|}{ Skeletal muscle results at rest } \\
\hline PGC-1 $\alpha$ mRNA (arbitrary units) & $1.02 \pm 0.12$ & $0.86 \pm 0.44$ & $0.99 \pm 0.24$ & $0.76 \pm 0.16$ \\
\hline MnSOD (U/mg) & $1.10 \pm 0.37$ & $1.53 \pm 0.51^{*}$ & $0.9 \pm 0.2$ & $1.33 \pm 0.39^{*}$ \\
\hline \multicolumn{5}{|l|}{ Constant work load exercise data } \\
\hline Work rate (W) & $86 \pm 14$ & $57 \pm 25^{* * *}$ & $72 \pm 20$ & $50 \pm 14^{* *}$ \\
\hline $\mathrm{V}^{\prime} 0_{2}$ peak $(\mathrm{l} / \mathrm{min})$ & $1.55 \pm 0.14$ & $1.13 \pm 0.26^{* *}$ & $0.83 \pm 0.19$ & $0.65 \pm 0.15^{*}$ \\
\hline $\mathrm{V}_{\text {epeak }}^{\prime}(1 / \mathrm{min})$ & $61.3 \pm 5.7$ & $44.6 \pm 5.9^{* *}$ & $36.7 \pm 9.1$ & $27.9 \pm 8.5^{*}$ \\
\hline End exercise dyspnoea, CR10 & $5.0 \pm 2.2$ & $4.1 \pm 1.4$ & $3.6 \pm 1.8$ & $2.0 \pm 1.0$ \\
\hline End exercise leg fatigue, CR10 & $7.5 \pm 1.8$ & $6.8 \pm 1.6$ & $4.7 \pm 2.1$ & $2.4 \pm 1.5^{*}$ \\
\hline Total $\mathrm{V}^{\prime} \mathrm{O}_{2}$ (mmol) & $2122 \pm 280$ & $1515 \pm 227^{* * *}$ & $1113 \pm 223$ & $865 \pm 187^{* * *}$ \\
\hline Estimated ROS production $(\mathrm{mmol} / \mathrm{kg})$ & $0.76 \pm 0.15$ & $1.45 \pm 0.24 * * *$ & $0.4 \pm 0.1$ & $0.81 \pm 0.16^{* * *}$ \\
\hline $\mathrm{PaO}_{2}$ peak exercise $(\mathrm{mm} \mathrm{Hg})$ & $80 \pm 4$ & $66 \pm 6^{* * *}$ & $87 \pm 5$ & $67 \pm 7^{* * *}$ \\
\hline Lactate end $(\mathrm{mEq} / \mathrm{l})$ & $5.4 \pm 0.7$ & $5.0 \pm 0.6$ & $2.4 \pm 0.3$ & $2.8 \pm 0.5$ \\
\hline
\end{tabular}

Values shown are mean \pm SD.

${ }^{*} \mathrm{p}<0.05$; ${ }^{* *} \mathrm{p}<0.01 ;{ }^{* * *} \mathrm{p}<0.001$ vs controls.

BMI, body mass index; CR10, Borg CR10 scale; FEV ${ }_{1}$, forced expiratory volume in $1 \mathrm{~s}$; FFMI, fat-free mass index; LT, lactate threshold; MnSOD, manganese superoxide dismutase; mtDNA mitochondrial DNA; nDNA, nuclear DNA; $\mathrm{PaO}_{2}$, arterial oxygen tension; $\%$ ref, percentage of predicted values; PGC- $1 \alpha$, peroxisome proliferator-activated receptor- $\gamma$ coactivator- $1 \alpha$; ROS, reactive

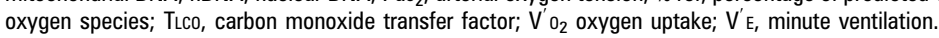


forward and reverse primers employed were 5'-CACTTACAAGCCAAACCAA

CAACT-3' and 5'-CAATAGTCTTGTTCTCAAATGGGGA-3', respectively. Values were expressed relative to the mean data obtained in controls at rest before exercise.

\section{Oxidative stress}

The mtDNA oxidation assay used is based on two premises ${ }^{16}$ : (1) oxidative DNA damage blocks the progression of polymerase and causes decreased DNA amplification; and (2) the probability of oxidative lesions is lower in shorter DNA segments. Thus, the ratio between the amplification of a short $(8.9 \mathrm{~kb})$ and a long (207 kb) fragment (relative amplification; RA) is a marker that mirrors mtDNA oxidation (ie, the lower RA value the higher mtDNA oxidation ${ }^{16}$ ). The two mtDNA fragments amplified were for the $8.9 \mathrm{~kb}$ fragment: mt5999F (5-TCTAAGCCTCC TTATTCGAGCCGA-3) and mt14841R (5-TTTCATCATGCGGAGATGTTGG ATGG-3) and the 207 bp fragment: mt4181F (5-ACTTCCTACCACTC ACCCTA-3) and mt4388R (5-TGATAGGTGGCACGGACAAT-3). PCR was performed as described above and the mean resting value of RA in control subjects was considered the reference value (100\% RA).

The amount of ROS produced during exercise was estimated assuming that, in healthy subjects, $2 \%$ of mitochondrial $\mathrm{V}^{\prime} \mathrm{O}_{2}$ is diverted into the generation of $\operatorname{ROS}^{17}$ and that this is 2.5 times greater in patients with COPD. ${ }^{8}$

Finally, the activity of MnSOD in skeletal muscle biopsies was measured by the inhibition of nitroblue tetrazolium conversion by MnSOD into a blue tetrazolium salt method (Sigma-Aldrich, St Louis USA), as described by Beauchamp and Fridovich. ${ }^{18}$

\section{Statistical analysis}

The results are presented as mean $\pm S D$ unless specified otherwise. Comparisons between groups were performed using the paired t test or two-way ANOVA with two factors (group and time), as appropriate. Contrasts at specific time points were made by the unpaired t test. Correlations between variables of interest were explored with the Pearson linear correlation coefficient. A $p$ value $<0.05$ was considered significant.

\section{RESULTS}

The main results are summarised in table 1. Age, body mass index and free fatty mass index were similar between groups. Airflow limitation was moderate to severe in patients with COPD whereas spirometry was normal in controls. Patients with COPD had moderate hypoxaemia. Aerobic capacity $\left(\mathrm{V}^{\prime} \mathrm{O}_{2}\right.$ peak) was normal in controls and moderately reduced in patients with COPD (table 1). Resting MnSOD concentrations were significantly higher in patients with COPD than in controls (table 1). At rest, when all the patients with COPD were compared with all controls, mtDNA/nDNA and PGC- $1 \alpha$ values were significantly lower in the former $(\mathrm{mtDNA} / \mathrm{nDNA}$ : $1096 \pm 196$ vs $1236 \pm 189, p=0.039 ;$ PGC $-1 \alpha$ : $0.081 \pm 0.33$ vs $1.01 \pm 0.17, p=0.035)$. However, the differences did not reach statistical significance when analysed separately by groups according to the specific type of constant exercise test to which they had been allocated (table 1).

\section{Exercise above LT}

To complete a total of $45 \mathrm{~min}$ pedalling at $65 \%$ of their $\mathrm{V}^{\prime} \mathrm{O}_{2}$ peak, six patients had to stop twice and three patients three times (all because of leg fatigue) whereas three completed the test at once without stopping. Likewise, four controls stopped once (because of leg fatigue) and six did it without stopping. No patient or control subject reached their $\mathrm{V}^{\prime} \mathrm{O}_{2}$ peak at any time during the constant work rate exercise challenge. Lactate levels after exercise were similar in both groups (table 1) and indicate that the intensity of the effort was well above the LT (table 1).

mtDNA/nDNA declined after exercise both in controls and patients with COPD but the changes were larger $(p=0.02)$ in patients with COPD (figure 1, upper panel). As a result, immediately after exercise mtDNA/nDNA had decreased by $-246 \pm 99$ in patients with COPD compared with $-167 \pm 49$ in controls $(p=0.036)$, whereas these figures were, respectively, $-424 \pm 147$ vs $-277 \pm 661 \mathrm{~h}$ after exercise $(p=0.009)$ and $-367 \pm 218$ vs $-194 \pm 11024 \mathrm{~h}$ later $(\mathrm{p}=0.036)$. One week after exercise (figure 1, upper panel) $\mathrm{mtDNA} / \mathrm{nDNA}$ had recovered baseline values in both groups ( $12 \pm 91$ vs $42 \pm 105, p=0.55)$. By contrast, the concentration of $\mathrm{MnSOD}$ tended to increase after exercise in parallel in both groups (figure 2, upper panel), the changes reaching statistical significance $24 \mathrm{~h}$ after exercise $(p=0.011$ and $p=0.014$ for patients with COPD and control subjects, respectively) and returning to baseline levels 1 week later. During this time course, differences in MnSOD observed at rest between patients and controls groups were basically unchanged throughout (figure 2, upper panel).

PGC- $1 \alpha$ mRNA levels at rest (table 1 ) tended to be reduced in patients with COPD $(p=0.07)$. After exercise (figure 3 , upper panel) they increased in both groups to a similar extent (figure 3 upper panel), but the changes were significantly higher $(\mathrm{p}=0.038)$ in patients with COPD $1 \mathrm{~h}$ after exercise (figure 3 , upper panel). By $24 \mathrm{~h}$ of recovery, PGC- $1 \alpha$ mRNA levels were repressed and corresponded to levels observed at rest (figure 3 , upper panel).

\section{Exercise below LT}

The lactate concentration at the end of exercise did not reach $3 \mathrm{mEq} / \mathrm{l}$, confirming that the intensity of exercise was below the LT (table 1). As shown in figure 1 (lower panel), low intensity exercise also induced significant changes in $\mathrm{mtDNA} / \mathrm{nDNA}$ with respect to baseline in patients with COPD $(-109 \pm 94$

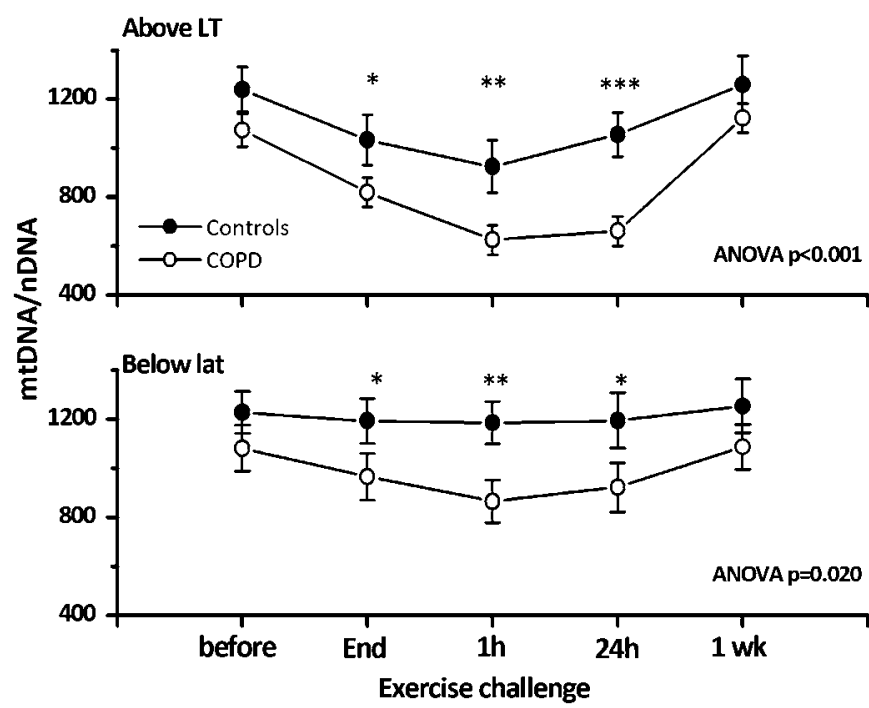

Figure 1 Time course of mean \pm SEM changes in mitochondrial DNA/ nuclear DNA ( $\mathrm{mtDNA} / \mathrm{nDNA}$ ) in controls (closed circles) and patients with chronic obstructive pulmonary disease (COPD) (open circles) exercising at high intensity (upper panel) and low intensity (lower panel). ${ }^{*} \mathrm{p}<0.05 ;{ }^{* *} \mathrm{p}<0.01 ;{ }^{* *} \mathrm{p}<0.001$ isotime COPD vs controls. LT, lactate threshold. 


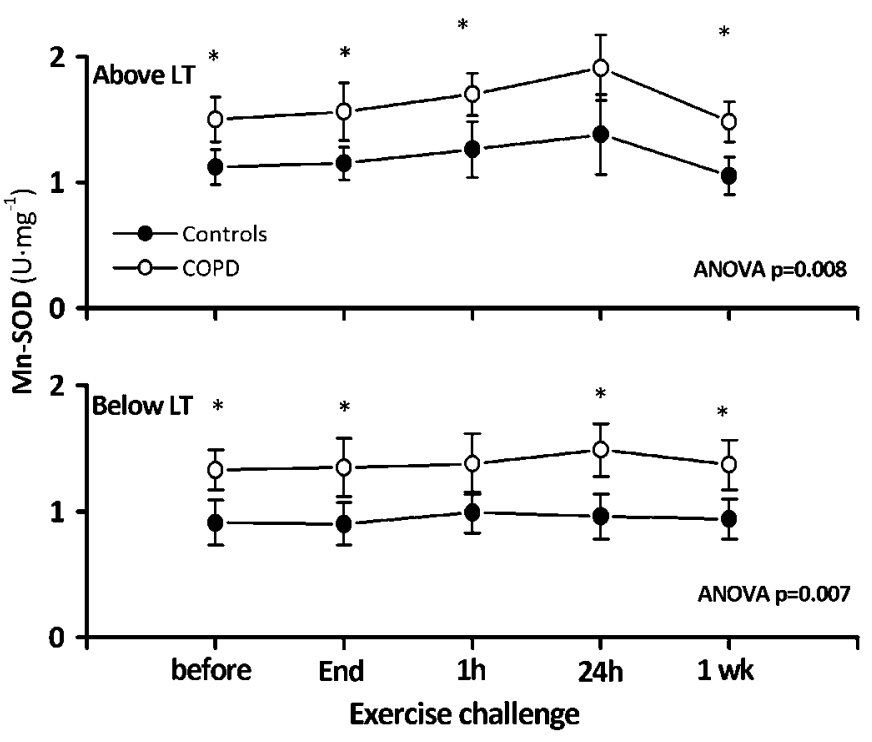

Figure 2 Time course of mean $\pm S E M$ changes in manganese superoxide dismutase (MnSOD) in controls (closed circles) and patients with chronic obstructive pulmonary disease (COPD) (open circles) exercising at high intensity (upper panel) and low intensity (lower panel). ${ }^{*} \mathrm{p}<0.05$ isotime COPD vs controls. LT, lactate threshold.

immediately after exercise, $\mathrm{p}=0.048 ;-220 \pm 911 \mathrm{~h}$ later, $\mathrm{p}=0.06$; and $-159 \pm 213$ after $24 \mathrm{~h}, \mathrm{p}=0.014)$, but not in controls $(-33 \pm 44, p=0.12$ immediately after exercise; $-73 \pm 205, p=0.38$ $1 \mathrm{~h}$ later; and $-33 \pm 94$ after $24 \mathrm{~h}, \mathrm{p}=0.39$ ). It should be noted also that absolute mtDNA/nDNA changes were smaller when patients with COPD exercise below the LT than above it $(p=0.007, p=0.024, p=0.004$ and $p=0.131$, respectively, for intergroup comparisons at the end of the exercise and $1 \mathrm{~h}, 24 \mathrm{~h}$ and 1 week later; figure 1). On the other hand, the MnSOD concentration was basically constant after exercise below the LT, so differences with controls were maintained through time (figure 2, lower panel) whereas changes in PGC-1 $1 \alpha$ mRNA were

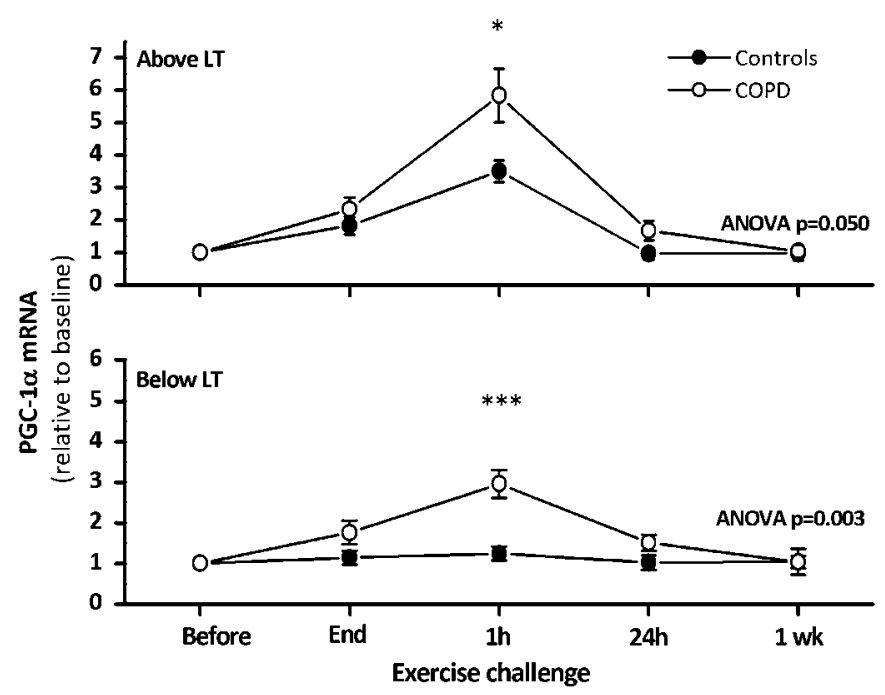

Figure 3 Time course of mean \pm SEM changes in peroxisome proliferator-activated receptor- $\gamma$ coactivator- $1 \alpha$ (PGC- $1 \alpha)$ in controls (closed circles) and patients with chronic obstructive pulmonary disease (COPD) (open circles) exercising at high intensity (upper panel) and low intensity (lower panel). ${ }^{*} p<0.05 ;{ }^{* * *} p<0.001$ isotime COPD vs controls. LT, lactate threshold. still evident in patients with COPD but absent in controls (figure 3, lower panel).

\section{Potential associations}

Because previous studies in healthy subjects ascribed a key pathogenic role to oxidative stress in the observed decrease in the mtDNA content in skeletal muscle, ${ }^{10}$ we explored potential relationships between the estimated production of ROS during exercise and changes from rest to $1 \mathrm{~h}$ after exercise in mtDNA/ nDNA in the entire population of subjects (patients and controls) studied both above and below the LT (figures 4 and 5). Our data confirm that a higher production of ROS during exercise was associated with a significant decrease in mRNA/ nDNA (figure 4A) and a larger change in MnSOD (figure 4C). Furthermore, we found a good correlation between the estimated ROS production and mtDNA relative amplification (figure 4B), suggesting that the former is a reasonably good proxy of the amount of oxidative stress during exercise.

On the other hand, we extended previous observations by showing that the estimated ROS production during exercise was also associated with a significant increase in PGC- $1 \alpha$ mRNA $1 \mathrm{~h}$ after exercise (figure 4D). Likewise, we observed significant correlations between the total amount of oxygen consumed per $\mathrm{kg}$ of fat-free mass during exercise and these same muscle variables (figure 5). Finally, we found that changes in PGC- $1 \alpha$ mRNA were also significantly related to changes in $\mathrm{mtDNA}$ / $n D N A(r=0.52, p<0.001)$ and MnSOD $(r=0.40, p=0.017)$ (data not shown).

\section{DISCUSSION}

Our results confirm that, in healthy subjects, fatiguing exercise induces a fall in skeletal muscle mtDNA content ${ }^{10}$ and upregulates PGC- $1 \alpha$ mRNA expression. ${ }^{14}{ }^{19}$ We extend these observations for the first time to patients with COPD where, as hypothesised, changes are enhanced (figures 1 and 3), probably in relation to augmented oxidative stress (figures 4 and 5). We also highlight the different recovery dynamics of PGC- $1 \alpha$ mRNA expression and mtDNA changes; the former returns to normal values $24 \mathrm{~h}$ after exercise (figure 3 ) whereas the latter takes longer to recover (figure 1). Our results also show that these effects are less marked when patients exercise below the LT (figures 1-3).

\section{Previous studies}

A number of cellular and molecular abnormalities have been described in the skeletal muscle of patients with COPD ${ }^{14} 2021$ including mitochondrial changes. ${ }^{1} 3589$ The lower PGC- $1 \alpha$ mRNA expression observed in skeletal muscle biopsies of patients with COPD at rest are in keeping with these previous observations and may be the consequence of inactivity or a switch towards type II fibres (known to express less PGC1 $\alpha$ mRNA and reported in COPD). ${ }^{1} 82021$ To our knowledge, however, this is the first study that has specifically investigated the effects of exercise, both below and above the LT, on these markers in these patients. Overall, the pattern of response to exercise of $\mathrm{mDNA} / \mathrm{nDNA}$ and PGC- $1 \alpha$ mRNA observed in patients with COPD is similar to that described in healthy subjects ${ }^{1014} 19$ but, for a given exercise intensity, mDNA/nDNA changes are magnified in COPD (figures 1 and 3 ).

\section{Potential mechanisms}

At rest the mtDNA/nDNA ratio was lower in patients with COPD. This is consistent with several abnormalities previously 

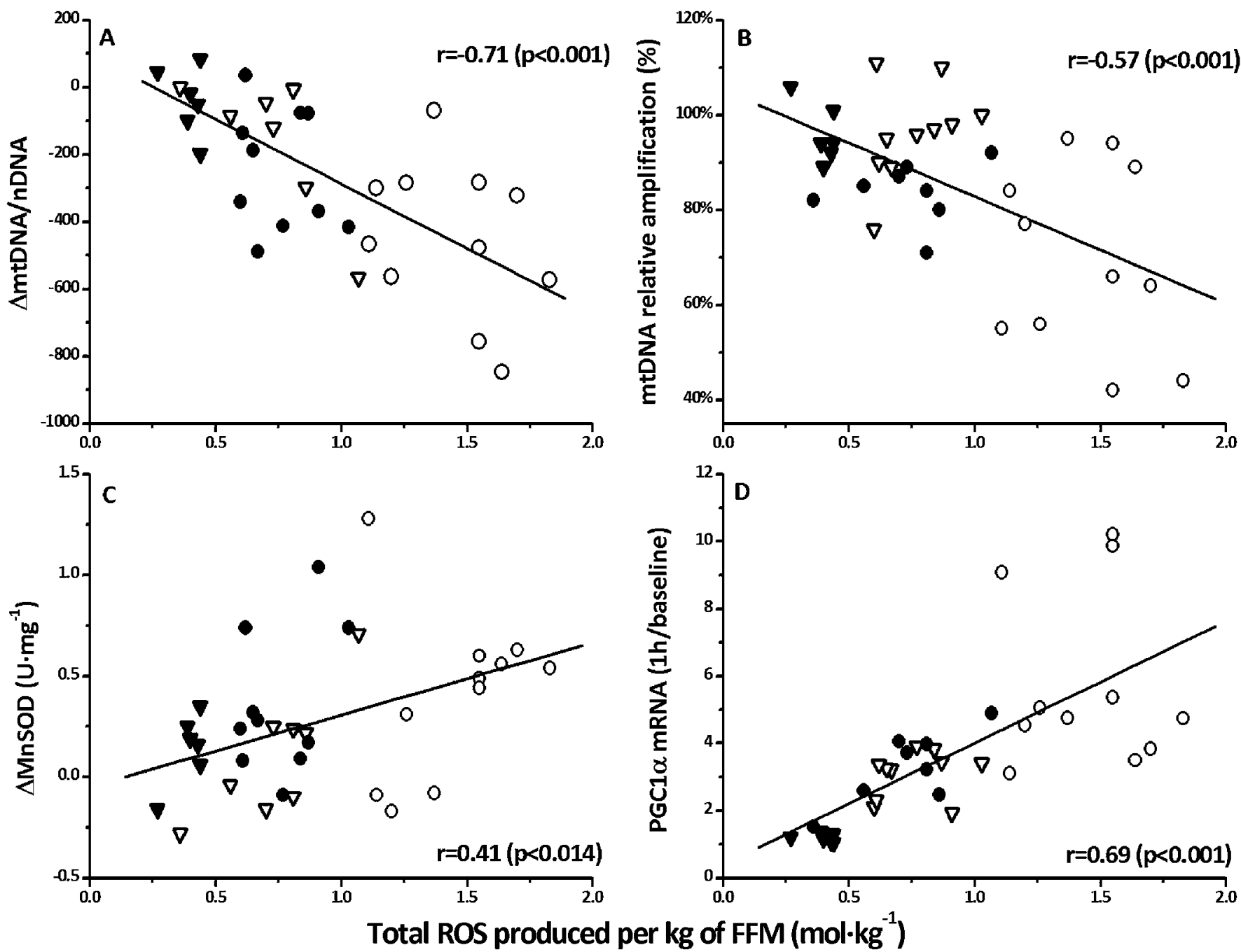

Figure 4 Correlation between estimated production of reactive oxygen species (ROS) during exercise and changes from rest to $1 \mathrm{~h}$ after exercise in (A) mitochondrial DNA/nuclear DNA (mtDNA/nDNA) ratio, (B) mtDNA oxidative damage (relative amplification), (C) manganese superoxide dismutase (MnSOD) and (D) peroxisome proliferator-activated receptor- $\gamma$ coactivator- $1 \alpha$ (PGC-1 $\alpha$ ) mRNA in controls (closed symbols) and patients with chronic obstructive pulmonary disease (COPD) (open symbols) studied both above (circles) and below the lactate threshold (triangles). FFM, fat-free mass.

described in these patients including decreased density of mitochondria in the vastus lateralis muscle, ${ }^{3}$ reduced citrate synthase activity ${ }^{4} 2122$ and/or a higher proportion of type II fibres ${ }^{1}$ with less oxidative capacity and lower PGC- $1 \alpha$-mRNA expression $^{23}$ (as was also observed in our patients).

We confirmed that the mtDNA content decreases in healthy subjects after fatiguing exercise ${ }^{1024}$ and we show for the first time that this is enhanced in patients with COPD. As previously suggested, ${ }^{10} 24$ our results support a pathogenic role of oxidative stress because: (1) it is well established that patients with COPD produce a larger amount of ROS than healthy controls during exercise ${ }^{11-13} 24$; (2) we found both temporal and statistical correlations between the estimated ROS burden produced during exercise and changes in mtDNA content (figure 4); (3) we observed a biological gradient between the expected ROS production and the changes in $\mathrm{mtDNA} / \mathrm{nDNA}$ that were reduced in patients with COPD exercising below their LT (figure 1) who also have less oxidative stress (table 1 and figures 4 and 5); (4) the fall in mtDNA after exercise was related to changes in MnSOD concentration and PGC- $1 \alpha$ mRNA content (figure 4), both known to be related to oxidative stress $^{22} 25$; and (5) it is biologically plausible that, as the main subcellular site of ROS production is the mitochondria, ${ }^{26} 27$ the concentration of ROS in the mitochondrial matrix is $5-10$-fold higher than in the cytosol or nucleus and the level of oxidised bases in mtDNA is $10-20$-fold higher than in DDNA. $^{28}$ In addition, unlike nDNA, mtDNA lacks protective histones ${ }^{26}$ and is endowed with relatively low DNA repair activity. ${ }^{26}$ It is therefore entirely plausible ${ }^{29}$ that enhanced ROS production during exercise in the skeletal muscle of patients with COPD may contribute to amplifying the decrease in mtDNA in these patients (figures 1 and 4). Together these arguments support a mechanistic role for oxidative stress in the enhanced decrease in mtDNA content in the skeletal muscle of patients with COPD after exercise.

\section{Clinical implications}

Our observations may be relevant for rehabilitation programmes in COPD because oxidative mtDNA damage and PGC- $1 \alpha$ mRNA expression are prerequisites for adaptation to training. ${ }^{10} 192230 \mathrm{In}$ this context it is noteworthy that moderate intensity exercise (below the LT) induced both a significant decrease in mtDNA/ nDNA and an increase in PGC- $1 \alpha$ mRNA expression in the patients with COPD. 
Figure 5 Correlation between measured total oxygen consumed during exercise and changes from rest to $1 \mathrm{~h}$ after exercise in $(\mathrm{A})$ mitochondrial DNA/nuclear DNA (mtDNA/nDNA) ratio, (B) mtDNA oxidative damage (relative amplification), (C) manganese superoxide dismutase (MnSOD) and (D) peroxisome proliferator-activated receptor- $\gamma$ coactivator- $1 \alpha$ (PGC-1 $\alpha)$ mRNA in controls (closed symbols) and patients with chronic obstructive pulmonary disease (COPD) (open symbols) studied both above (circles) and below the lactate threshold (triangles). FFM, fat-free mass.
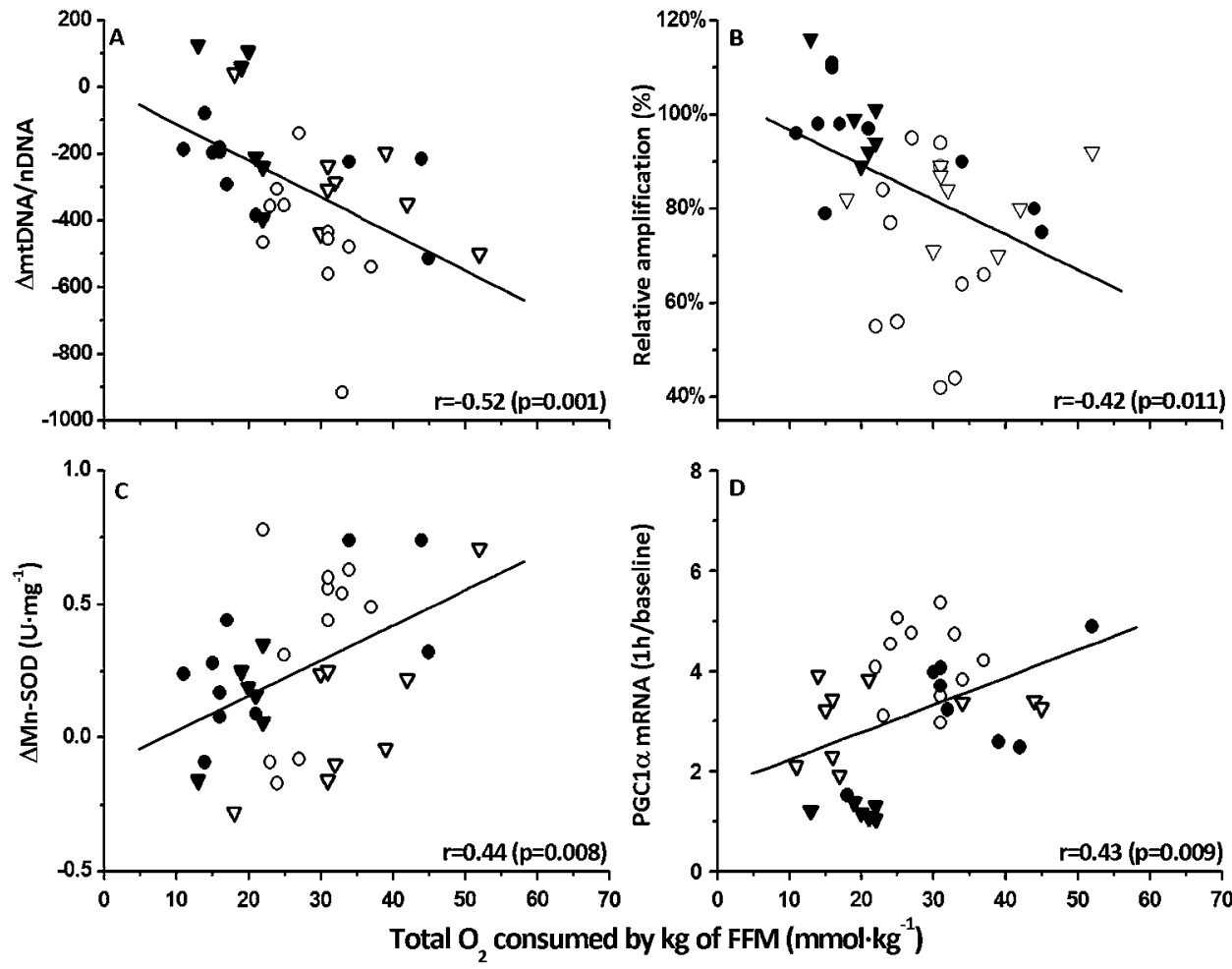

The response of PGC- $1 \alpha$ mRNA to exercise indicates that, at least in the patients with COPD studied here who were not characterised by a low body mass index (table 1), the first step for mitochondrial biogenesis and type I fibre differentiation is not altered $^{22}$ and that, consequently, other mechanisms should be implicated in the pathobiology of muscle atrophy in COPD. ${ }^{1} 31$ This observation also raises concerns about the potential utility of antioxidant supplementation during rehabilitation as they could dampen ROS signalling and interfere with mitochondrial biogenesis. $^{22}$

\section{Potential limitations}

Some potential limitations of our study deserve discussion. First, the sample size was relatively small and biopsy specimens were not available 1 week after exercise in a few subjects. However, we believe that our results are valid because they were internally consistent within each group. Second, different subjects participated in the two exercise protocols (below and above the LT) because the considerable number of biopsies involved in the study precluded other alternatives. However, the baseline characteristics of the patients and controls in both protocols were similar (table 1) and the biological response of healthy subjects to exercise, both above and below the LT, was similar to that previously described. Third, ROS production during exercise was not measured directly but was estimated from published data. ${ }^{8} 17$ However, we think that the correlation observed with several independent variables such as mtDNA/nDNA, MnSOD and oxidised mtDNA support our interpretation of the results. Finally, patients with COPD were treated with $\beta_{2}$ agonists but these drugs do not appear to influence mitochondrial biogenesis signals, including PGC-1 $\alpha^{32}$

\section{Conclusions}

Our study shows that exercising at the typical intensity and duration of training sessions in pulmonary rehabilitation programmes produces a significant decrease in the mtDNA content of skeletal muscle in patients with COPD and overexpression of PGC- $1 \alpha$ mRNA, probably in relation to enhanced oxidative stress. Interestingly, the changes are minimised but not abolished with non-fatiguing exercise below the LT, and this may be relevant for the training effect seen in these patients training below the LT.

Acknowledgements The authors thank the participants in this study for their willingness to contribute to the advancement of science. They are also grateful to Professor Antonio L Andreu and Professor Helena García-Arumí from the Centre d'Investigació en Bioquímica i Biologia Molecular, University Hospital Vall d'Hebron Barcelona, Spain for their help in the determination of the mtDNA/nDNA. CIBERES is an initiative of the Instituto de Salud Carlos III (Ministerio de Ciencia e Innovación).

Funding Supported by Fondo de Investigaciones Sanitarias (PS09/02391).

Competing interests None.

Patient consent All participants gave written informed consent after being made fully aware of the goals and potential risks of the study.

Ethics approval This study was conducted with the approval of the Committee for Ethics in Human Research of Madrid Sanitary Area 1 and all aspects of the study comply with the Declaration of Helsinki.

Provenance and peer review Not commissioned; externally peer reviewed.

\section{REFERENCES}

1. Anon. Skeletal muscle dysfunction in chronic obstructive pulmonary disease. A statement of the American Thoracic Society and European Respiratory Society. Am J Respir Crit Care Med 1999:159:S1-40.

2. Agusti AG, Sauleda J, Miralles C, et al. Skeletal muscle apoptosis and weight loss in chronic obstructive pulmonary disease. Am J Respir Crit Care Med 2002; 166:485-9.

3. Gosker HR, Hesselink MK, Duimel H, et al. Reduced mitochondrial density in the vastus lateralis muscle of patients with COPD. Eur Respir J 2007;30:73-9.

4. Maltais $\mathbf{F}$, LeBlanc $P$, Whittom $F$, et al. Oxidative enzyme activities of the vastus lateralis muscle and the functional status in patients with COPD. Thorax 2000;55:848-53

5. Rabinovich RA, Bastos R, Ardite E, et al. Mitochondrial dysfunction in COPD patients with low body mass index. Eur Respir J 2007;29:643-50.

6. Remels AH, Schrauwen P, Broekhuizen R, et al. Peroxisome proliferator-activated receptor expression is reduced in skeletal muscle in COPD. Eur Respir $\mathrm{J}$ 2007:30:245-52 
7. Sauleda J, Garcia-Palmer F, Wiesner RJ, et al. Cytochrome oxidase activity and mitochondrial gene expression in skeletal muscle of patients with chronic obstructive pulmonary disease. Am J Respir Crit Care Med 1998;157:1413-17.

8. Puente-Maestu L, Perez-Parra J, Godoy R, et al. Abnormal mitochondrial function in locomotor and respiratory muscles of COPD patients. Eur Respir $J$ 2009;33:1045-52.

9. Puente-Maestu L, Perez-Parra J, Godoy R, et al. Abnormal transition pore kinetics and cytochrome $C$ release in muscle mitochondria of patients with chronic obstructive pulmonary disease. Am J Respir Cell Mol Biol 2009:40:746-50.

10. Marcuello A, Gonzalez-Alonso J, Calbet JA, et al. Skeletal muscle mitochondrial DNA content in exercising humans. J Appl Physiol 2005;99:1372-7.

11. Couillard A, Koechlin C, Cristol JP, et al. Evidence of local exercise-induced systemic oxidative stress in chronic obstructive pulmonary disease patients. Eur Respir J 2002;20:1123-9.

12. Mercken EM, Gosker HR, Rutten EP, et al. Systemic and pulmonary oxidative stress after single-leg exercise in COPD. Chest 2009;136:1291-300

13. van Helvoort HA, Heijdra YF, de Boer RC, et al. Six-minute walking-induced systemic inflammation and oxidative stress in muscle-wasted COPD patients. Chest 2007; 131:439-45

14. Holloszy J0. Regulation by exercise of skeletal muscle content of mitochondria and GLUT4. J Physiol Pharmacol 2008;59(Suppl 7):5-18.

15. Rabe KF, Hurd S, Anzueto A, et al. Global strategy for the diagnosis, management, and prevention of chronic obstructive pulmonary disease: GOLD executive summary. Am J Respir Crit Care Med 2007;176:532-55.

16. Santos $\mathbf{J H}$, Mandavilli BS, Van HB. Measuring oxidative mtDNA damage and repair using quantitative PCR. Methods Mol Biol 2002;197:159-76.

17. Chance B, Sies H, Boveris A. Hydroperoxide metabolism in mammalian organs. Physiol Rev 1979:59:527-605.

18. Beauchamp C, Fridovich I. Superoxide dismutase: improved assays and an assay applicable to acrylamide gels. Anal Biochem 1971;44:276-87.

19. Pilegaard H, Saltin B, Neufer PD. Exercise induces transient transcriptional activation of the PGC-1alpha gene in human skeletal muscle. J Physiol 2003;546:851-8.

20. Gosker HR, Bast A, Haenen GR, et al. Altered antioxidant status in peripheral skeletal muscle of patients with COPD. Respir Med 2005:99:118-25.
21. Puente-Maestu L, Tena T, Trascasa C, et al. Training improves muscle oxidative capacity and oxygenation recovery kinetics in patients with chronic obstructive pulmonary disease. Eur J Appl Physiol 2003;88:580-7.

22. Vina J, Gomez-Cabrera MC, Borras C, et al. Mitochondrial biogenesis in exercise and in ageing. Adv Drug Deliv Rev 2009;61:1369-74.

23. Lin J, Wu H, Tarr PT, et al. Transcriptional co-activator PGC-1 alpha drives the formation of slow-twitch muscle fibres. Nature 2002:418:797-801.

24. Pinho RA, Chiesa D, Mezzomo KM, et al. Oxidative stress in chronic obstructive pulmonary disease patients submitted to a rehabilitation program. Respir Med 2007:101:1830-5.

25. Silveira LR, Pilegaard $\mathrm{H}$, Kusuhara $\mathrm{K}$, et al. The contraction induced increase in gene expression of peroxisome proliferator-activated receptor (PPAR)-gamma coactivator 1alpha (PGC-1alpha), mitochondrial uncoupling protein 3 (UCP3) and hexokinase II (HKII) in primary rat skeletal muscle cells is dependent on reactive oxygen species. Biochim Biophys Acta 2006:1763:969-76.

26. Cadenas E, Davies KJ. Mitochondrial free radical generation, oxidative stress, and aging. Free Radic Biol Med 2000:29:222-30.

27. Giulivi C, Boveris A, Cadenas E. Hydroxyl radical generation during mitochondrial electron transfer and the formation of 8-hydroxydesoxyguanosine in mitochondrial DNA. Arch Biochem Biophys 1995:316:909-16.

28. Richter C, Gogvadze V, Laffranchi R, et al. Oxidants in mitochondria: from physiology to diseases. Biochim Biophys Acta 1995;1271:67-74.

29. Shokolenko I, Venediktova N, Bochkareva A, et al. Oxidative stress induces degradation of mitochondrial DNA. Nucl Acid Res 2009;37:2539-48.

30. Wang H, Hiatt WR, Barstow TJ, et al. Relationships between muscle mitochondrial DNA content, mitochondrial enzyme activity and oxidative capacity in man: alterations with disease. Eur J Appl Physiol Occup Physiol 1999;80:22-7

31. Mercken EM, Hageman GJ, Langen RC, et al. Decreased exercise-induced expression of NF-KB regulated genes in muscle of COPD patients. Chest Published Online First: 2010 Aug 5. doi:10.1378/chest.10-0275

32. Robinson MM, Richards JC, Hickey MS, et al. Acute $\beta$-adrenergic stimulation does not alter mitochondrial protein synthesis or markers of mitochondrial biogenesis in adult men. Am J Physiol Regul Integr Comp Physiol 2010;298:R25-33.

\section{Lung alert}

\section{Lactate clearance is not inferior to central venous oxygen saturation as a goal in early sepsis resuscitation}

Goal-directed therapy in severe sepsis improves outcome by providing protocol-driven targets for initial resuscitation. Current guidelines use central venous oxygen saturations $\left(\mathrm{ScvO}_{2}\right)$ to assess oxygen delivery and metabolism, but this requires specialist equipment and expertise which may limit its use. This study addresses whether a change in serum lactate concentration may act as an alternative.

This prospective multicentre trial randomised 300 patients with severe sepsis or septic shock to one of two resuscitation protocols. Initially, central venous pressure and mean arterial pressure were normalised using crystalloid boluses and vasopressors. Red blood cell transfusion and dobutamine were then used to either achieve a target $\mathrm{ScvO}_{2}$ of $>70 \%$ or a lactate clearance of $10 \%$ within the first $2 \mathrm{~h}$ of resuscitation.

There was no significant difference in absolute in-hospital mortality between the two groups (34 patients (23\%) died in the $\mathrm{ScvO}_{2}$ group vs $25(17 \%)$ in the lactate clearance group). There was no difference in treatment-related adverse events. Only 29 patients received a blood transfusion or dobutamine to achieve these targets.

This study shows that lactate clearance is a simple but effective measure of total body oxygen metabolism which may be comparable to $\mathrm{ScvO}_{2}$ when resuscitating patients with severe sepsis. However, this study was not blinded and was performed in hospitals with established goal-directed sepsis protocols and hence may not be generalisable to other institutions.

- Jones AE, Shapiro NI, Trzeciak S, et al. Lactate clearance versus central venous oxygen saturation as goals of early sepsis therapy: a randomized clinical trial. JAMA 2010:303:739-46.

\section{Amelia Dunscombe}

Correspondence to Amelia Dunscombe, Bristol Royal Infirmary, Bristol BS2 8HW, UK; ameliadunscombe@yahoo.co.uk

Published Online First 14 October 2010

Thorax 2011;66:127. doi:10.1136/thx.2010.145607 